\title{
A New Paradigm for Analysing Knowledge Transfer Processes*
}

\author{
Mikhail Oseledchik \\ Department of Philosophy and Social Sciences \\ High school of printing and media industry \\ Moscow Polytechnic University \\ Moscow, Russia \\ balu13@yandex.ru
}

\author{
Marina Ivleva \\ Department of Social Philosophy \\ Peoples' Friendship University of Russia \\ Moscow, Russia \\ marinanonna@yandex.ru
}

\author{
Vitaly Ivlev \\ National Research University \\ MSTU named after N.E. Bauman \\ Moscow, Russia \\ vitalijivlev@yandex.ru
}

\begin{abstract}
The paper considers knowledge as one of the most important and explored concepts in the history of philosophy, and in particular the logic, epistemology and methodology of science. The proposed paper focuses on new approaches to the analysis of knowledge and knowledgetransfer processes, which, in the author's opinion, can be accepted as a new modern paradigm for the analysis of these concepts. At the heart of this paradigm lie three fundamental ideas-the interpretation of knowledge as a fractal; representation of knowledge as a non-scale network; and analysis of knowledge as a non-equilibrium dynamic system.
\end{abstract}

Keywords-knowledge; knowledge transfer; paradigm; fractal; non-scale network; non-equilibrium dynamic system

\section{INTRODUCTION}

Knowledge is one of the most important and explored concepts in the history of philosophy and, in particular, the logic, epistemology and the methodology of science. Nevertheless, because of the complexity and multidimensionality of the object under investigation, a clear definition of the concept of 'knowledge' has not been developed.

The proposed article is considering new approaches to the analysis of knowledge and the processes of knowledge transfer, which, in the author's opinion, can be accepted as a principle in the new modern paradigm of analyzing these concepts.

At the heart of this paradigm, there are three fundamental ideas:

*This paper was financially supported by the Ministry of Education and Science of the Russian Federation on the program to improve the competitiveness of Peoples' Friendship University (RUDN University) among the world's leading research and education centers in the 2016-2020 (The Agreement number 02.A03.21.0008).
- Interpretation of knowledge as a fractal;

- Representation of knowledge as a non-scale network;

- Analysis of knowledge as a non-equilibrium dynamic system [1].

Without repeating more than once the author's reviews of approaches to the analysis of knowledge let us turn to the consideration of these ideas [2] [3].

\section{USING THE CONCEPT OF 'FRACTAL' TO REVEAL THE ESSENCE OF KNOWLEDGE}

In the general sense, knowledge is the result of human cognitive activity, which acts as assimilated concepts, laws, principles, fixed images of phenomena and objects.

In our work, we use the concept of 'fractal' to interpret the essence of knowledge. To begin with, the ideal model for transferring knowledge in the process of training or in the process of communication (whether in business, in science or simply in interpersonal communication) is the translation of the total amount of information units, understanding their meanings and relationships from the source of the original knowledge, to its recipient. In reality, we have a completely different picture - the recipient assimilates only a part of knowledge, its fragments, he does not see a whole series of interrelations, does not understand the true meaning of the transmitted information, constantly sees in the acquired knowledge what, in the opinion of the carrier, cannot be present in it; the recipient introduces a lot of additional information units and their meanings that he invents on his own initiative.

Anger and complaints about this are completely meaningless, since the classical models of knowledge transfer at the level of instinct base on the interpretation of 
translation and the assimilation of knowledge as a simple linear process.

In other words, if the carrier transmits to the addressee knowledge of the explanatory type: A \& BVA \& $\urcorner_{\mathrm{B}} \rightarrow \mathrm{C}$, we hope that the recipient will learn exactly the same $A$ \& BVA \& $\urcorner_{7} \mathrm{~B} \rightarrow \mathrm{C}$ formula. However, in reality, we see the recipient can reconfigure this formula as D \& BVAVB $\rightarrow$ $\mathrm{CV}_{7} \mathrm{C}$.

In fact, the transfer of knowledge is not a simple linear process since the consciousness of the recipient of knowledge is not a pure board on which you can rewrite the original formula. The recipient of knowledge already has its own ready-made system of explicit and implicit knowledge, his own stock of meanings, his life experience, a certain level of language proficiency, the level of intellectual development and other important personal characteristics. This system, at every stage of a person's life, is balanced and develops dynamically. Therefore, the receipt of new information leads to violation of this equilibrium; new information may be a foreign body for this established system of a person's inner world, which, frankly, most often happens. Hence, we must first understand that the system of human knowledge is the result of the process of self-organization and it lives by nonlinear laws, so its behavior often becomes unpredictable, which can be the source of the unexpected reconfiguration of knowledge, which is both good and bad at the same time. It is bad because it is almost impossible to transfer knowledge accurately and in full. It is good, because it gives creative reconfiguration and increment of knowledge.

Then, in order to achieve the assimilation of knowledge, that is, the reproduction of the knowledge of the carrier in the system of the recipient's knowledge in the most complete and accurate manner, i.e. like it is in the original form, we can use the interpretation of knowledge as a fractal - a selfsimilar structure, whose image does not depend on scale, as a recursive dynamic model, each part of which repeats the development of the whole model in its development.

Considering that both the carrier and the recipient of knowledge each have their own system of knowledge, and that the knowledge of each individual is a self-organizing and self-similar structure, because both the carrier and the recipient of knowledge have a lot of common knowledge-a common system of values, common language, common lexical-semantic structure of this language, the standard logic of reasoning, ways and styles of thinking, common stock of conventional knowledge, skills and abilities, a sense of humor, even if all these are developed and assimilated with varying degrees, but nevertheless all of them are similar to each other because both the carrier of knowledge and the recipient exist within the limits of the same socio-cultural paradigm - we can say: the knowledge has a fractal nature.

Therefore, we can state that knowledge is a stochastic fractal.

- Knowledge of the individual is conceptual, it is a fractal in relation to the conceptosphere of society as a whole.
- The assimilation of knowledge by the individual and its reconfiguration is influenced by a number of attractors, the number, power and directivity of which are practically impossible to trace. Hence, the possible reconfiguration and production of new knowledge is almost impossible to calculate.

- Knowledge of the individual is almost impossible to examine: to enumerate all units, the entire content of person's knowledge is unrealistic due to his partial unconsciousness by the person himself. The totality of the individual's knowledge is enormous, but it is in a folded non-actualized form (e.g. the surface of a person's lungs is larger than a tennis court, but it is squeezed into a very limited volume, the same phenomenon is characteristic of the human circulatory system, here can be mentioned Maldenbrot's favorite example of the practically endless coastline of Great Britain). Depending on the requirements of adaptation to these environmental conditions at any particular time, it turns out that the person has some more knowledge. Since knowledge is conceptual, the intellectual units of its nucleus are more or less examined, but peripheral knowledge, largely possessing the characteristics of fuzzy sets, is codified only partially.

- Knowledge of an individual can be systematized only partially, depending on the possibility of its fundamental codification; the rest of the array of knowledge is contained in the form of unencoded elements, in particular, in the form of practical skills and abilities, intuitions, metaphors, certain emotional images, but at the same time, all these elements of knowledge are closely interconnected through numerous semantic series, associative, logical and other chains, which, if necessary, allows instantaneous actualization of necessary knowledge.

- Knowledge is dialogical-it is always a product of the internal and external dialogue of the knowledge carrier with its addressee in case of knowledge transfer and in the process of its individual rethinking. Consequently, knowledge of an individual never remains static and equilibrium: there is a constant process of generating and degenerating of knowledge, its rethinking and reinterpretation, complication and primitivisation as a result of the external and internal dialogue of the knowledge carrier.

- Knowledge is communicative - in the process of communication, it is more accurately assimilated by the individual; for the more effective transfer of his knowledge, the person also reconfigures it, turning it into a more systematized, accurate and understandable for the addressee.

- Assimilation and understanding of the acquired by the person knowledge depends on the level of discipline and training of his thinking, on the general level of culture and education, on the standards of the accepted logic, on the social orientation of the person, 
on the impact on the process of assimilation of the sets of attractors. Consequently, the depth and correctness of understanding is not the result of a linear process of consuming knowledge. We can never with a high degree of accuracy perceive the knowledge of another individual due to a number of small circumstances that influence the way of understanding and revealing certain semantic units. At the same time, the interpretation of knowledge as a stochastic fractal allows us to be loyal to the fact that the knowledge conveyed by the carrier, which consists of the elements $\{\mathrm{ABCDEF}\}$, will be reproduced as a self-similar system \{A1B1C1D1E1F1\}.

- The ability to reconfigure the acquired knowledge and its increment by the person depends on the degree of his creativity and the level of freedom from the usual cultural schematisms in favor of new inversion options (in favor of new logic and new schematisms and the choice of a new way of semantic formation - this was mentioned by K. Levi-Strauss and V.S. Bibler), so it is almost impossible to calculate and predict new results and discoveries that a particular individual will make. However, with a high degree of probability, it is possible to predict the possibility that a particular subject is capable of obtaining new results and discoveries, and can make a good career.

- There is a constant development of mental abilities of the subject, the totality of his experience, the emergence of new influential attractors or the change in the degree of influence of already existing ones, which leads to the change in and rethinking of the set of knowledge available to him.

- In the knowledge of the subject there are erroneous elements, often the subject can use absurd ways of substantiating judgments that can give both true and false results.

Consequently, the transfer of knowledge is, at first, the process of breaking the fractal, because while receiving new knowledge it destroys the existing cognitive system of the recipient and is perceived and interpreted by him incompletely and inaccurately. Then, due to the selfsimilarity of the cognitive systems of different individuals who live and think in a common lexical-semantic space, the process of assembling the fractal begins - the obtained constituent parts of new knowledge are combined into unified formulas and are perceived more or less adequately and isomorphically to the original message. New knowledge of the recipient turns into fractal knowledge of the addressee. Then comes the most interesting and important stage-the process of knowledge re-configuration, when, thanks to the presence of a whole set of hard-to-recognize personal attractors of the recipient, knowledge begins to be reinterpreted and rethought. The result of this reconfiguration is a creatively enriched knowledge, often unexpected in its new content for its original carrier.
The assimilation of these new meanings of knowledge, in turn, entails the restructuring of the system of knowledge of the carrier and its transformation into the fractal of the recipient's knowledge. Moreover, this, in turn, leads to the transformation of the entire cognitive paradigm, which unites both the carrier and the recipient of knowledge.

Now we need to pay attention to another important detail If knowledge is a self-organizing, complex and evolving system, the notion of 'management' can be applied to it with a great deal of convention: management is always the introduction of organizational changes; it turns out that we organize a self-organizing system, which sounds absurd.

We come to the most fundamental moment-the difference in the transfer of knowledge in the process of training and communication.

Any training is a transfer of knowledge from an 'instructor' to a 'student', i.e. from the bearer of new knowledge, who owns a larger fragment of general culture as an ordered semiotic field, to the addressee who uses a smaller fragment of the cultural space. In this case, the addressee undergoes a targeted cultivation of the cognitive fractal, in accordance with the already existing cognitive fractal of the knowledge carrier.

In the process of communication, the transfer of knowledge proceeds from one peer-to-peer user of a cultural semiotic space to another user. In this case, we are dealing with the understanding and assimilation of new knowledge as with the process of assembling the fractal.

In the process of communication, there is an exchange of not only codified, but also of the so-called 'implicit', personal knowledge. Therefore, in order to optimize this process of fractal assembly, the cultural semiotic space must be to the maximum extent saturated with meanings and perception of the fractal elements should be facilitated through a single sense-formation system. To do this one needs to involve all the abilities of human thinking and the sensory perception of the world. This is why the use of metaphors, analogies, stories, visual images, intuitions, musical fragments - everything that initiates processes of perception and generation of meanings and changes the emotional state of a person, which ultimately leads to the fact that suddenly there comes a moment insight —all parts of the puzzle fall into place, and there is a moment of full understanding of new knowledge.

\section{REPRESENTATION OF KNOWLEDGE AS A NON-SCALE NETWORK}

The second idea, underlying the new paradigm of knowledge analysis, is the representation of knowledge as a non-scale network [4].

We use for this purpose the methodology of V.V. Balashov, A.V. Smirnov and T.O. Tseitlina [5].

For knowledge and knowledge-sharing system, the following properties of non-scale graphs are characteristic:

- The presence of 'strongly connected concentrators'. They have a large number of connections and connect 
the network together, forming the so-called 'concentrator-like nucleus'. This creates a stable, but at the same time, a vulnerable to the targeted attacks structure of connections, which is a fundamental sign of non-scale networks". Such concentrators include language as a means of forming, storing and transferring knowledge, logic as a criterion for the correctness of constructing the system of inferences, a worldview system that determines the basic content of knowledge of community, general cultural knowledge, which retains its significance over long periods of time, mathematical apparatus, a system of symbolic records.

- The degree distribution of the number of connections between nodes of the network-the greater the number of direct links $\mathrm{k}$ at the concentrator, the fewer concentrators with the same number of direct links in the network we have (say, the language of communication - the so-called natural language spoken by the members of this community, has a lot more direct ties (because with the help of its means existing knowledge is expressed, which in principle allows its codification), than in the specialized language of logic, mathematics or programming)).

- Self-similarity of the network: complex networks have a certain hierarchy of nodes. At each level of the hierarchy of knowledge and knowledge management there are tightly connected clusters, therefore the system of knowledge of each individual can be considered as a non-scale network and part of the global knowledge system (say, the basis for any system and individual and general knowledge are concepts of the structure of the world and society).

- Small network diameter, one of the important network parameters, is the 'network diameter' - the maximum length of the shortest paths between any two nodes of the network (because of the categorical nature of our thinking, which is expressed in the fact that we think with the help of generalized images, genders of objects and phenomena, transitions from one area of knowledge to another with the help of associations, analogies and metaphors occur almost instantaneously, for example, we can easily make an analogy between the cellular structure of plants and the atomic structure of molecules and in principle all bodily formations, or take analogy between human brain and a computer logic structure).

- Mechanisms of forming and developing network. The formation of new links in the network does not occur in a random manner, but in accordance with the mechanism of preferential accession: the new elements are mainly attached to the hub-nodes having more connections. In addition, the second mechanism of forming links in a non-scaled network is assortativity, a preference in forming links within a group of nodes compared to the forming links external to this group, which contributes to the enhancement of clustering in a growing non-scale network. Thanks to these two mechanisms -preferential accession and assortativity - a non-scale network retains its properties regardless of size. An example is the rapid development of research in the field of genetic engineering, the explosion of Internet technology, the use of Max Planck's quantum ideas, the theory of relativity by Albert Einstein or the theory of 'scaleless networks' of A. Barabashi to explain the world order [6]. As soon as there appears a new conceptual element of knowledge which can explain a multitude of phenomena, this element becomes an increasingly more important concentrator thanks to the mechanisms of preferential attachment and assortativity.

Thus, we have every right to view knowledge as a non-scale network.

\section{ANALYSIS OF KNOWLEDGE AS A NON-EQUILIBRIUM DYNAMIC SYSTEM}

The third idea is the analysis of knowledge as a nonequilibrium dynamic system. It is necessary here to enter a reservation that this is not a tribute to the fashionable synergetic tradition.

Knowledge is always an open system of information exchange with the environment - an individual exchanges information in the process of communication with other individuals, and knowledge as such, being a mix of assertive, negative and hypothetical components, constantly changes, acquiring new elements of knowledge, by confirming its hypothetical components, by reassessing and rejecting its assertive components, which get the status of obsolete and erroneous ones, by expanding the number of negative components. The process of self-organization characterizes such a system. This process is nonlinear, the development of knowledge is ambiguous, multivariant, and the pace of its development is constantly changing.

Using the terminology and ideology of I. Prigozhin and S N. Kurdyumov, we can regard knowledge as a dissipative system, in which entropy constantly increases [7] [8]. In fact, knowledge as a whole is a unity of knowledge of the individual and knowledge of the society, which possesses a codified core and a huge, fundamentally uncodifiable peripheral content, defined by the intellectual, psychological and everyday circumstances of the individual's being. The distribution of knowledge among individuals is completely uneven and asymmetrical, as well as the principal possibilities for the growth of individual knowledge.

Thus, the intensity of development of individuals' knowledge and their various social groups is fundamentally different. Assimilation of general knowledge by weaker individuals and marginal social groups leads to its disorganization, since these bearers of knowledge are characterized by ignorance and fragmentary perception of cognitive units. On the other hand, stronger individuals and their communities are faced with a constant increase in the flow of information that blurs the established orderly structures of the knowledge organization. Nevertheless, the general knowledge system is able to digest and organize the 
resulting chaotic avalanches of new knowledge according to the structures of attractors existing within the knowledge system (these are sets of the most stable structures, the forms to which the processes in dissipative environments evolve, for instance, such structures-attractors are the traditional forms of developing knowledge, for example, a problem, a hypothesis and a theory in the form of which the new knowledge is fixed). Consequently, knowledge as a whole can be regarded as a self-sustaining, self-organizing structure. As S. N. Kurdyumov pointed out, "an ordered structureprocess grows out of chaos, and chaos, in turn, takes the process out to one of the structures-attractors: it generates order."

This is the new paradigm of analyzing knowledge that we propose.

\section{CONCLUSION}

We offer a fundamentally new approach to the analysis of the concept of 'knowledge' and of the processes of knowledge transfer. This new paradigm bases on three key ideas:

Knowledge is a stochastic fractal.

Knowledge and the system of knowledge transfer is a non-scale network.

Knowledge is a non-equilibrium dynamic system.

This approach gives a fundamentally new understanding of the essence of knowledge and the processes of knowledge transfer.

\section{REFERENCES}

[1] M. B. Oseledchik, V.Y. Ivlev, M.L. Ivleva, Knowledge as a nonequilibrium dynamic system, Proceedings of the 2017 2nd International Conference on Contemporary Education, Social Sciences and Humanities (ICCESSH 2017), part of the series ASSEHR, Moscow, V/124, pp. 1-5.

[2] M. B. Oseledchik, Logical-philosophical aspects of knowledge management: monograph / M.B. Oseledchik. Moscow: Moscow State University named after Ivan Fedorov, 2011. Chapter 2, paragraph 2.2, p. 244.

[3] M. B. Oseledchik, V. A. Inozemtsev, V. Y. Ivlev, M. L. Ivleva, Logical-philosophical approach to the interpretation of the concept of knowledge // Proceedings of the 2016 3rd International conference on education, language, art and inter-cultural communication (ICELAIC 2016). Advances in social science, education and humanities research, Paris: Atlantis Press, 2017, vol. 40, pp. 297-301.

[4] M. B. Oseledchik, Logical-philosophical aspects of knowledge management: monograph / M.B. Oseledchik, Moscow: Moscow State University named after Ivan Fedorov, 2011, p. 244.

[5] V. V. Balashov, Consideration of the network of Russian domestic long-distance airlines as a non-scale graph [Electronic resource] / V. V. Balashov, A.V. Smirnov, T. O. Tseitlina. - Access mode: http://readings.gmik.ru/files/2010/965a02d856dd34d7b60f4de5654d4 6ff.pdf//.- 24.07.2011.

[6] A. -L Barabashi, Non-scalable networks / A.-L. Barabashi, E. Bonabo // In the world of science, 2003, No. 8, pp. 55-63.

[7] I. Prigozhin, I. Stengers, Order from chaos / I. Prigozhin, Moscow, 1986, p. 432.
[8] E. N. Knyazeva, S. N. Kurdyumov, Foundations of synergetics: a man constructing himself and his future / E. N. Knyazeva, M .: KomKniga, 2010, p. 232. 